(abstract). The sudden onset of monoclonic jerks mainly involving the trunk and upper limbs, normal developmental milestones, absence of EEG abnormalities and self limited course were consistent with the diagnosis as described initially by Lombroso and Fejerman.

\title{
AMANTADINE IN ABSENCE EPILEPSY
}

Refractory absence epilepsy in 4 children was treated with amantadine as an add-on drug at the Tel Aviv University Sackler School of Medicine, Israel. The patients responded within 1 week and remained free of symptoms for 27-36 months without adverse effects. The dosage was $5-7 \mathrm{mg} / \mathrm{kg}$ per day given twice a day up to a maximum of $200 \mathrm{mg}$ daily (Shahar EM, Brand N. Effect of add-on amantadine therapy for refractory absence epilepsy. $\underline{J}$ Pediatr of Neurol Nov 1992; 121:819-821). (Reprints: Eli M. Shahar, M.D., Child Neurology Unit, Chaim Sheba Medical Center, Tel Hashomer 52621, Israel.)

COMMENT. Amantadine was initially used by Shields et al. in the treatment of 10 children with minor motor seizures refractory to medications. Myoclonic seizures and atypical absence seizures responded well (Neurology $1985 ; \underline{35: 579)}$ ).

\section{VALPROATE AND EEG ABNORMALITIES}

The effect of valproate on EEG epileptic abnormalities was investigated at the Institute of Neurophysiology, Genoa, Italy. Sixteen patients aged 14 to 44 years were administered a single $(14-37 \mathrm{mg} / \mathrm{kg})$ oral dose of magnesium valproate. At peak serum concentrations of $65-139 \mathrm{ug} / \mathrm{ml}$ of valproate, there were increases in spike wave frequency at $2,3,4$ and 5 hours. The peak concentrations of ammonia ranged from 15-72 ug/1, with values exceeding normal in 13 determinations. The VPA and ammonia concentrations varied independently. The frequency of EEG epileptic abnormalities was not correlated with either the ammonemia level or the amount of variation in ammonemia concentration (Sannita WG. Valproate acute administration, EEG epileptic abnormalities, and ammonemia. Neurology Oct 1992; 42:20032005). (Reprints: Professor Walter G. Sannita, Centro Farmaci Neuroattivi, Dipartimento di Scienze Motorie, Universita, Ospedale San Martino, viale Benedetto XV, I-16132 Genova, Italy.)

COMMENT. A direct CNS drug action has been proposed to explain the quantitative EEG abnormalities in response to acute VPA administration and the stupor states in some patients. The stupor was not necessarily related to the increased ammonia concentration in the blood.

\section{BRAIN NEOPLASMS}

\section{BRAIN RADIOTHERAPY AND COGNITION}

Cognitive function and school achievement were studied prospectively over 3 to 4 years in 19 children treated for brain tumors with whole-brain 